Results In total, 310 participants were included in the study (response $70 \%$ ). The majority were men who have sex with men. Seventy five percent of the study population was referred directly from STI clinic to treatment centre by a clinician or nurse. Thirty percent of participants were delayed: $15 \%$ entered care after 4 weeks and $15 \%$ had not entered care yet. Thus, $70 \%$ of participants entered care within 4 weeks; median time of entry was 9 days (range 0-739 days). In the multivariate model, age below 25 years ( $\mathrm{OR}=3.695 \%$ CI 1.9-6.7) and indirect referral (OR $=3.395 \%$ CI 1.8-6.5) were independent risk factors for care delay, while people with health insurance were less likely to delay ( $\mathrm{OR}=0.395 \%$ CI $0.1-0.9)$. No association was found between care delay and CD4 cell counts or viral load at diagnosis.

Conclusions Younger persons and persons without health insurance are at risk for entering care late after being diagnosed HIV positive. Direct referral from STI clinic to care leads to less delay. Testing of those at risk is not enough to interrupt HIV transmission, entry into care needs to be assured as well. A more active role of the care provider is needed.

\section{P2.154 DETERMINANTS OF UTILIZATION OF A NO-COST HIV TRANSITION CLINIC IN UGANDA: A CROSS SECTIONAL STUDY OF YOUNG ADULTS LIVING WITH HIV/AIDS}

doi:10.1136/sextrans-2013-051184.0418

A Nyabigambo. Makerere University, College of Health Sciences, School of Public Health, Kampala, Uganda

Objective The main purpose of this study was to understand the levels and determinants the HIV Transition Clinic services utilisation by young adults at Infectious Diseases Institute, Kampala, Uganda.

Methods A cross sectional study using quantitative methods at a HTC in a sample of 379 young adults living with HIV/AIDS (YALHA) between the ages of 15-24 years. At analysis utilisation was categorised into two levels; regular (kept all appointment visits) and irregular (missed one or more appointments visits) utilisation. Univariable, bivariable and multivariable logistic regression was used to establish determinants associated with utilisation of the HTC.

Results Of the 379 total respondents, only $32.4 \%$ were regular utilizers of the HTC. There are low levels of regular utilisation of the HTC. Female young adults have better service utilisation rates compared to the males in HTC. The male to female ratio was 1:5. The determinants of HTC regular utilisation were CD4 cell count category of 250-2603/ $\mu \mathrm{l}$ (AOR 0.58, 95\% CI: 0.36-0.95), not currently on ART (AOR 0.27, 95\% CI: 0.15-0.47) and did not receive counselling services (AOR 0.47, 95\% CI: 0.27-0.83).

Conclusion The factors that were associated with reduced the chance of regular use of the HTC were: CD4 cell count between 250-2603/ $\mu \mathrm{l}$; not being on ART; and not receiving HIV counselling services.

Recommendations The young adults in the HIV transition clinic should be screened on a regular basis to detect those with a CD4 cell count of $<350 / \mu \mathrm{l}$ and counselled for early initiation of ART so as to enhance regular HIV transition clinic utilisation. In addition a reminder system like peers, family and mobile phone messages should be set up for young adults so that they are reminded of their appointments hence facilitating regular use of the transition clinic.

\section{P2.155 ADHERENCE MATTERS: A PATIENT-CENTERED ADHERENCE STRATEGY TO ACHIEVE MAXIMUM HEALTH AND QUALITY OF LIFE OUTCOMES FOR PERSONS LIVING WITH HIV/AIDS IN PLAINFIELD, NEW JERSEY}

doi:10.1136/sextrans-2013-051184.0419

L Kudryashova Hernandez. Neighborhood Health Services Corporation, Plainfield, NJ, United States
Background Neighborhood Health Services Corporation (NHSC), an urban community-based not-for-profit ambulatory primary care health centre located in Plainfield, New Jersey, USA, provides HIV care and treatment services to over 350 uninsured, impoverished and minority persons living with HIV/AIDS. To achieve long-term positive health and quality of life outcomes, it is imperative that patients adhere to HIV care and treatment, as well as to prescribed medication regimens.

Methods At NHSC patient-centred adherence efforts begin with a personalised interview. An assessment of patient's clinical and lifestyle indicators is conducted by clinicians followed by formal adherence education video presentations. Other key components of the adherence strategy are detailed discussions of antiretroviral therapies and potential side effects. Patients are provided with culturally and linguistically sensitive printed materials regarding medications and potential side effects. Free Medication boxes are also provided. Weekly monitoring phone calls are conducted by clinical staff to assess compliance and identify adherence challenges. If patients miss appointments, confidential phone calls are placed to reschedule. Treatment Education and Adherence Record is a comprehensive tool that is administered annually to assess adherence, compliance, barriers and any unanticipated outcomes. A multi-disciplinary clinical team led by an Infectious Disease Specialist observes the entire process and monitors patients' boosted compliance with the project and promotion of overall HIV treatment and medications adherence.

Results Resulting from the patient-centred adherence strategy, NHSC observed: $25 \%$ improvement in medical visits no-show rates; $10 \%$ decrease in hospitalisation rates. Furthermore, $62 \%$ of patients maintained CD4 above 200; $52 \%$ of patients have achieved and maintained undetectable viral load.

Conclusions The collected and analysed data suggests that patient-centred adherence efforts help to: reduce no-show rates; minimise side effects and hospitalizations related to missed doses and "drug holidays"; improve and sustain optimal clinical indicators, as well as significantly improve health and quality of life outcomes.

\section{P2.156 PLACE OF NURSES IN THE MANAGEMENT OF PEOPLE LIVING WITH HIV AND AIDS (PLHA)IN DEVELOPING COUNTRIES: CASE OF A REGIONAL HOSPITAL, BURKINA FASO (WEST AFRICA)}

doi:10.1136/sextrans-2013-051184.0420

'Y Sagna, ${ }^{2} \mathrm{M}$ Mano, ${ }^{1,3} \mathrm{~J}$ Y Drabo. ${ }^{1}$ Internal Medicine Department of Yalgado Ouédraogo University Hospital (CHU YO), Ouagadougou, Burkina Faso; ${ }^{2}$ Medicine Department of Tenkodogo Regional Hospital, Tenkodogo, Burkina Faso; ${ }^{3}$ African network of training on HIV (RAF-VIH), Ouagadougou, Burkina Faso

Background Management of people living with HIV and AIDS (PLHA) in the Tenkodogo regional hospital was carried out by a team of 7 nurses and 4 physicians engaged also about their function in the management of non-HIV-infected patients. Only one of these nurses had already received specific training (inter-university graduate) on the management of HIV infection. This team had to manage actually 1213 PLHA including 505 under antiretroviral treatment (ART). Objective We aimed to describe the activities carried out by nurses of this team in the management of PLHA.

Methods We retrospectively reviewed all consultation activities for PLHA by the 7 nurses of the HIV-infected patient's management team at the Tenkodogo regional hospital from January to June 2011. The daily consultation registers of these nurses were our data sources.

Results 2709 consultations of PLHA (any reason confused) were carried out. The consultations mean was 451.5/month. 1886 ART renewals prescription have been made, including 1767 first line and 119 second line treatment. 44 PLHA were newly treated by ART 
during the study period. 708 (26.1\%) cases of diseases frequently associated with HIV were carried out. Suspected and treated pathologies were among other simple malaria in 262 cases (37\%), 177 cases of pulmonary diseases (25\%) including 03 cases of tuberculosis, 109 cases of gastroenteritis acute (15.4\%), 29 oral candidiasis and 29 sexual transmitted infections ( $4.1 \%$ each) and 4 cases of Herpes Zoster $(0.6 \%)$. For these diseases, the treatment success rate was $95.5 \%$. 16 hospitalizations have been made and 16 cases of consultations have been referred to physicians.

Conclusion The nurse can be a resource used to deal with the lack of physicians in management of PLHA especially if he received a specific training. This management training should be a key point in its basic studies curriculum in developing countries.

\section{P2.157 THE EXPERIENCE OF IMPLEMENTING POINT-OF-CARE HIV TESTING IN GP PRACTICES IN THE UNITED KINGDOM}

doi:10.1136/sextrans-2013-051184.0421

'S K Brooks, ${ }^{2}$ W Hachmoller. ${ }^{1}$ College of Agricultural Sciences and Education, Port Antonio, Portland, Jamaica; ${ }^{2}$ NHS North East London and the City, Newham Public Health Directorate, London, UK

Background NHS Newham implemented a General Practitioner (GP) rapid Point-of-care (PoCT) pilot for the testing of HIV in 2010. This was in response to recommendations made by the UK Chief Medical Officer and the British HIV Association for the expansion of routine HIV testing in all health care settings.

Methods Under the 12 month pilot, ten GP practises in Newham were recruited and trained to conduct rapid HIV testing, using the INSTI HIV-1/HIV-2 rapid testing kit. An HIV test was opportunistically offered to existing and new patients as routine. Accepting patients were tested following a pre-test discussion. Reactive antibody tests were referred to HIV clinical services.

Results During the pilot, a total of 698 tests were conducted in the participating GP practises. Of those tested, $58 \%$ were female and $37 \%$ were male. Eighteen percent (18\%) of clients tested were Black African. Greater up-take of HIV testing took place in the 25-34 year old age group. There were 11 reactive test results and 5 indeterminate results.

Conclusions Point-of-care (PoCT) testing of HIV is compatible in GP practises and acceptable to patients. Strategies are required for sustaining and expanding Point-of-care HIV testing in GP practises in London.

\section{P2.158 ONE-STOP SHOP SERVICE DELIVERY MODEL: INTEGRATING PREVENTION INTERVENTIONS WITH HIV CARE/ TREATMENT SERVICES IN A COMMUNITY-BASED MEDICAL HOME SETTING}

doi:10.1136/sextrans-2013-051184.0422

L Kudryashova Hernandez. Neighborhood Health Services Corporation, Plainfield, NJ, United States

Background Neighborhood Health Services Corporation (NHSC), an urban community-based not-for-profit ambulatory health centre in Plainfield, New Jersey, USA, provides services to over 350 persons living with HIV/AIDS. Presently $75 \%$ of NHSC HIV patients have history of substance abuse, $62 \%$ have mental health issues and/or depression and $30 \%$ are at risk for homelessness. For these patient populations long-term health and quality of life outcomes can not be achieved and sustained without aggressive intervention around substance use, mental health and other contributing factors.

Methods NHSC incorporates a coordinated, patient-centred approach to integrating multiple prevention services and interventions with HIV primary care and treatment in a welcoming, nonthreatening environment of a patient-centred medical home.
Substance abuse and mental health screenings are done by clinicians upon patient enrollment and every six months thereafter. Referrals for in-depth substance abuse and mental health assessments are generated as needed or at a minimum annually per clinical protocols. Patients in need of these services receive on-going counselling and appropriate interventions on-site. Referrals are also made to offsite facilities for crisis intervention and inpatient services. Psychosocial, financial and lifestyle assessments are conducted every six months to assess patients' risk for homelessness, substance use and unsafe lifestyle practises.

Results Resulting from an integrated, patient-centred approach to providing HIV services NHSC demonstrated the following: $95 \%$ of HIV patients received substance abuse and mental health screening; $100 \%$ received medical case management assessments. Furthermore, 57 patients receive on-going substance abuse counselling; 36 patients receive mental health counselling; 16 persons are in shelter/ transitional housing; 2 persons were hospitalised for suicide prevention.

Conclusions Integration of prevention interventions with HIV care/treatment under the umbrella of Early Intervention Services allowed to: achieve improved understanding of the reality of substance abuse/mental health; establish a seamless one-stop shop service delivery model and improve patients' access to community prevention/treatment resources.

\section{P2.159 KNOWLEDGE AND PRACTICE OF NURSES IN THE PREVENTION OF VERTICAL TRANSMISSION OF HIV IN SELECTED HOSPITAL OF EASTERN REGION OF NEPAL}

doi:10.1136/sextrans-2013-051184.0423

N Pokharel. B.P. Koirala institute of health sciences Dharan,Nepal, Dharan, Nepal

Vertical transmission remains the main mode of acquisition of HIV infection in children. A total of 700,000 children were newly infected in 2003, mainly through mother-to-child transmission of HIV. ${ }^{1}$ Mother to child transmission is also the largest source of HIV infection in children in Nepal. Nurses having the knowledge of HIV can bring positive changes in behaviour. Knowledge, training and experience in every aspect of one's profession are very important.

Objective The objective of this study is to assess knowledge and practise of Nurses in the Prevention of vertical transmission of HIV. Methods \& Materials:

Study Design A cross sectional study was done including 112 nurses drawn from the three selected hospital (BPKIHS Dharan, Koshi Zonal hospital Biratnagar and Mechi Zonal Hospital, Bhadrapur,) through population enumeration method.

Results The study show that half $(50.8 \%)$ of the respondents had adequate knowledge and near half (49.1\%) had inadequate level of knowledge, where total mean score \pm SD $28.3 \pm 5.5$. Only $16.1 \%$ of the respondents had good practise for prevention of vertical transmission of HIV. Qualification and place of working area are associated with knowledge of HIV/AIDS and PMTCT, where P value is $0.006,0.001$ respectively.

Conclusion In General, adequate knowledge was found half $(50.8 \%)$ of the respondents. Thus, more educational programmes should be focused on increasing their knowledge about vertical transmission, hoping to overcome the misconceptions that may be help to behaviour change toward safer practises.

\section{P2.160 UNDERAGE SEX - WHO CARES?}

doi:10.1136/sextrans-2013-051184.0424

A Delamere, A King, F Mulcahy. GUIDE Clinis, St James's Hospital, Dublin, Ireland

Background The Young Persons Clinic (YPC) was set up within the GUIDE Clinic as a dedicated clinic for young people aged 